\title{
Protective role of parnaparin in reducing systemic inflammation and atherosclerotic plaque formation in ApoE-/- mice
}

\author{
MARCO ARTICO $^{1}$, RACHELE RIGANÒ ${ }^{2}$, BRIGITTA BUTTARI ${ }^{2}$, ELISABETTA PROFUMO ${ }^{2}$, BRUNELLA IONTA ${ }^{1}$, \\ SANDRO BOSCO $^{3}$, MANUELA RASILE $^{2}$, ENRICA BIANCHI $^{1}$, MOIRA BRUNO $^{1}$ and LORENZO FUMAGALLI ${ }^{4}$ \\ ${ }^{1}$ Department of Sensory Organs, University of Rome 'Sapienza'; ${ }^{2}$ Department of Infectious, Parasitic and Immuno-mediated \\ Diseases of the Superior Health Institute of Rome; Departments of ${ }^{3}$ Experimental Medicine and ${ }^{4}$ Anatomical, \\ Histological, Medico-legal and Locomotor System Sciences, University of Rome 'Sapienza', Rome, Italy
}

Received November 15, 2010; Accepted December 29, 2010

DOI: $10.3892 / \mathrm{ijmm} .2011 .606$

\begin{abstract}
Atherosclerosis is a degenerative disease whose role in the onset and development of cardiovascular pathologies and complications is of importance. Due to its silent but progressive development, and considering the endothelial, immunological and inflammatory processes that are involved in its clinical course, this still relatively unknown pathological condition has been and continues to be a matter of investigation worldwide. Our experience with previous studies on atherosclerosis led us to investigate the possible influence of a low molecular weight heparin (LMWH) - Parnaparin ${ }^{\circledR}$ on the development and clinical course of atherosclerosis in double knock-out laboratory animals (ApoE-/- mice). Our experiments demonstrated a possible role of Parnaparin (PNP) in the control of atherogenic disease. In fact, in treated mice vs. untreated ones, PNP reduced the number and the size of atherosclerotic lesions in the aortic wall, as well as the development of liver steatosis, which was massive in untreated animals and moderate in treated ones. These preliminary observations require further clinical studies, but demonstrate a possible role of Parnaparin in the control of the development and clinical evolution of atherosclerosis and liver steatosis in laboratory animals.
\end{abstract}

\section{Introduction}

There is increasing evidence that inflammation plays an important role in atherosclerosis and its clinical complications, namely cardiovascular disease including coronary heart disease and ischaemic stroke (1). Despite this growing evidence $(2,3)$ the precise molecular and cellular mechanisms underlying thrombotic events remain poorly understood.

Correspondence to: Professor Marco Artico, Department of Sensory Organs, University of Rome 'La Sapienza', Viale del Policlinico 155, 00161 Rome, Italy

E-mail: marco.artico@uniroma1.it

Key words: inflammation, atherosclerosis, ApoE-/- mice, liver steatosis, Parnaparin
Unstable atherosclerotic plaques are vulnerable to rupture, and usually contain an increased accumulation of inflammatory cells, particularly macrophages and T-lymphocytes secreting growth factors (EGF and bFGF) and pro-inflammatory cytokines (4-6). Rather than remaining localized within the 'culprit' plaque, the inflammatory components spread throughout the peripheral blood. Several studies suggest an association between pro-inflammatory cytokines (TNF $\alpha$, IL-6, IL-1) in the peripheral blood of patients with atherosclerosis and the clinical outcome or stage of atherosclerotic disease (7-11). Despite the advances in interventional techniques, the management of stable atherosclerosis remains the domain of optimal guideline-oriented therapy (12). Most pharmacological interventions attempting to improve endothelial dysfunction target the risk factors linked to atherosclerosis: hypertension (ACE-inhibitors, calcium antagonists), dyslipidaemia (lipidlowering agents) and menopause (estrogens). Nevertheless, several pharmacological agents have been suggested for achieving vascular protection through different mechanisms beyond their primary therapeutic actions. These include ACE-inhibitors, statins, third generation beta-blockers (nebivolol), endothelium-derived nitric oxide synthesis (tetrahydrobiopterin, BH4) and antioxidant agents (13). Recent data suggest that unfractionated heparin (UFH) and low-molecular weight heparins (LMWH), that are well-defined anticoagulant agents (14-16), may also possess potential antiinflammatory properties; however, the mechanism underlying their anti-inflammatory effect has not been fully elucidated. The anti-inflammatory properties of heparin have been demonstrated in animal studies and in several clinical trials for the treatment of inflammatory bowel disease, arthritis and asthma. Furthermore, in vitro studies have shown that UFH and heparin derivates down-regulate pro-inflammatory cytokine expression (17-19) in human mononuclear cells, probably by inhibiting $\mathrm{NF}-\kappa \mathrm{B}$ activation $(14,20,21)$. A previous study demonstrated that Parnaparin (also known as Fluxum), an LMWH, significantly prevented L-selectin shedding, tissue factor expression and degranulation by polymorphonuclear leukocytes (22). This LMWH is efficient and generally well tolerated in the prevention of venous thrombosis and in the treatment of chronic venous disease and venous and arterial thrombosis (23). To our knowledge, no studies have defined 
the role of Parnaparin in preventing or reducing the onset or progression of atherosclerotic lesions.

The aim of our experimental study was to evaluate a possible functional role of Parnaparin in reducing vascular and systemic inflammation, and atherosclerotic lesion development in a mouse model. For this purpose, ApoE-/- double knock-out mice treated and untreated with Parnaparin were fed a high-fat diet (Western diet), and the extent of atherosclerotic lesions was evaluated along the aorta and in the aortic sinus at sacrifice by a histochemical procedure.

\section{Materials and methods}

Parnaparin. Parnaparin ${ }^{\circledR}$ was kindly provided by Alfa Wassermann S.p.A. Parnaparin sodium, an original product of Alfa Wassermann S.p.A. (24-28), is the sodium salt of a LMWH that is obtained by the radical-catalyzed depolymerization with hydrogen peroxide and with cupric salt, of heparin derived from porcine intestinal mucosa.

The majority of the components have a 2-O-sulpho- $\alpha-\mathrm{L}$ idopyranosuric acid structure at the non-reducing end and a 2-N,6-O-disulpho-D-glucosamine structure at the reducing end of their chain. The average molecular mass ranges between 4,000-6,000 with a characteristic value of about 5,000 (29) and the anti-Xa/anti-IIa ratio is between 1.5-3.0.

Animals. Targeted deletion of the gene for apolipoprotein E (ApoE knockout mice) leads to severe hypercholesterolemia and spontaneous atherosclerosis. Male ApoE-/- doubleknockout mice (18-20 g) were maintained on either the standard (A1, A2 and A3 groups: 20 animals) or Western diet (B1, B2 and B3 groups: 20 animals) for 18 weeks. The Western-type diet $(0.2 \%$ cholesterol, $42 \%$ fat) was purchased from Harlan Teklad, Inc. (Td 88137, Indianapolis, IN). Both groups of mice were further divided into the three following subgroups: control mice either untreated (groups A1 and B1, 5 animals) or subcutaneously injected with PBS (groups A2 and B2, 5 animals) and mice subcutaneously injected for six days/week with $5 \mathrm{mg} / \mathrm{kg}$ of Parnaparin in PBS (groups A3 and B3, 10 animals). Mice were weighed weekly to assess the exact Parnaparin amount to be injected to reach the optimal drug concentration. The Parnaparin concentration used to treat ApoE-/- mice was chosen on the basis of preliminary experiments on C57BL/6 mice that represent the wild-type mice with the same genetic background of ApoE-/- mice. In these preliminary experiments, Parnaparin was injected daily at 1,5 and $10 \mathrm{mg} / \mathrm{kg}$ for a week to evaluate its possible toxic effects in different organs (liver, kidney, heart).

Before treatment and once a month thereafter, a serum sample was obtained from each mouse by retro-orbital plexus puncture for pro-inflammatory marker profile evaluation (data not shown). Animals were euthanasized by slow $\mathrm{CO}_{2}$ inhalation, 18 weeks after treatment. The heart, aorta, carotid arteries, liver and kidneys were removed from each mouse. No relevant alterations were observed in the different organs with the exception of two animals treated with $10 \mathrm{mg} / \mathrm{kg}$ Parnaparin, which developed a mediastinal haemorrhagic syndrome detected immediately after sacrifice. In light of the results of these preliminary experiments $5 \mathrm{mg} / \mathrm{kg}$ Parnaparin were chosen as the optimal dose used for animal treatment.
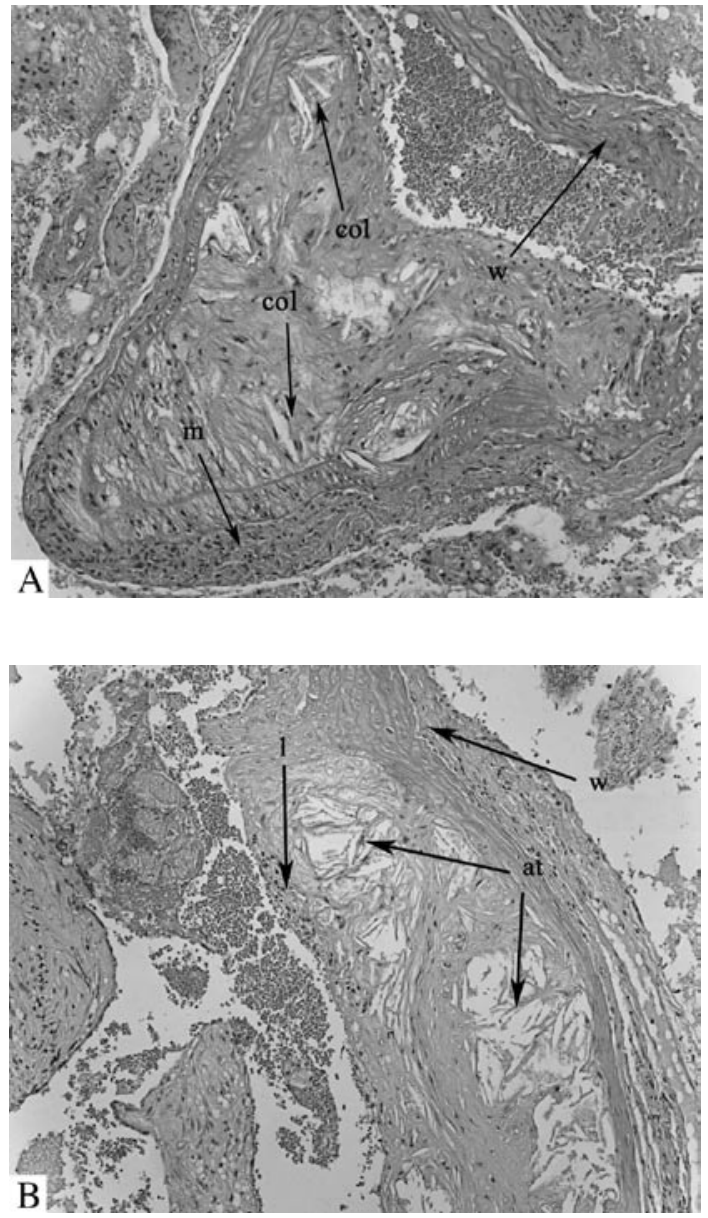

Figure 1. Animal control groups (A1 and B1) fed a standard diet (A) and a Western diet (B), and not treated with PNP (x20 magnification). The atherosclerotic lesions in the wall (w) of the aorta appeared marked, with typical cholesterol spindle-shaped deposits (col) in the sub-endothelial layer together with an inflammatory infiltrate with macrophages (m) and lymphocytes (1) around the atherosclerotic plaque (at).

Experiments were conducted according to the guidelines formulated by the European Community for experimental animal use (L358-86/609EEC), and were approved by the Italian Ministry of Health.

\section{Results}

Evaluation of atherosclerotic lesions. Atherosclerotic lesions were analyzed (optical microscopy) after H\&E staining was performed on specimens harvested from the aorta and liver of treated (PNP) and untreated (control) ApoE-/- mice. In the control mice, the atherosclerotic lesions in the wall $(w)$ of the aorta appeared marked, with typical cholesterol spindleshaped deposits (col) in the sub-endothelial layer together with an inflammatory infiltrate with macrophages $(\mathrm{m})$ and lymphocytes (1) around the atherosclerotic plaque (Fig. 1). A reduction of the atherosclerotic lesions (at) was observed in treated (PNP) animals by observing either the alteration of the arterial wall (w) or the extension of the atherosclerotic plaque. The latter appeared reduced in size and produced a less evident disarrangement of the morphology of the arterial wall (Fig. 2) in treated mice compared to the analogous lesions in untreated animals. Liver steatosis was also reduced 

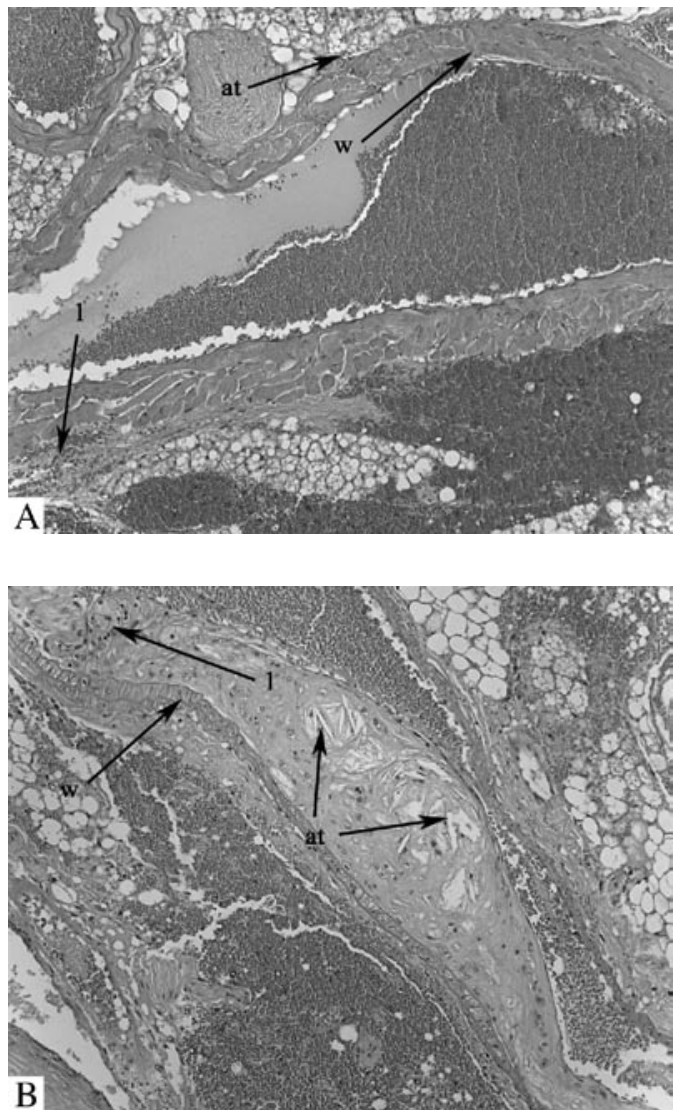

Figure 2. Animal groups (A3 and B3) fed a standard diet (A) and a Western diet (B), were treated with PNP (x20 magnification). A reduction of the atherosclerotic lesions (at), together with a less pronounced inflammatory infiltrate with lymphocytes (1), was observed in treated animals by observing the alteration of the arterial wall (w) and the extension of the atherosclerotic plaque (at). The latter appeared reduced in size and produced a less evident disarrangement of the morphology of the arterial wall in treated mice compared to the analogous lesions in untreated mice.

and appeared only moderate in treated (PNP) animals (Fig. 3), who developed a focal and, in some cases, microsteatosis in comparison to the massive, diffuse and macroscopic evidence of liver steatosis observed in untreated animals (Fig. 4).

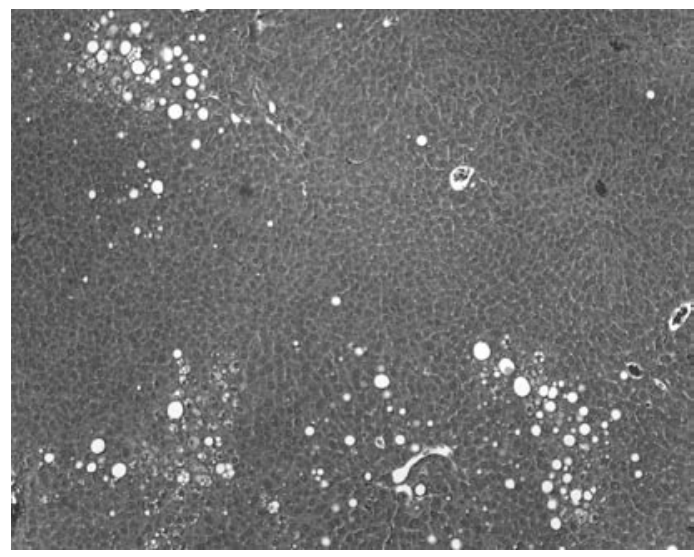

Figure 3. Focal liver microsteatosis developed in mice fed a Western diet, after treatment with PNP (x20 magnification). Liver steatosis was reduced and appeared only moderate in these animals, in comparison to untreated animals (Fig. 4)
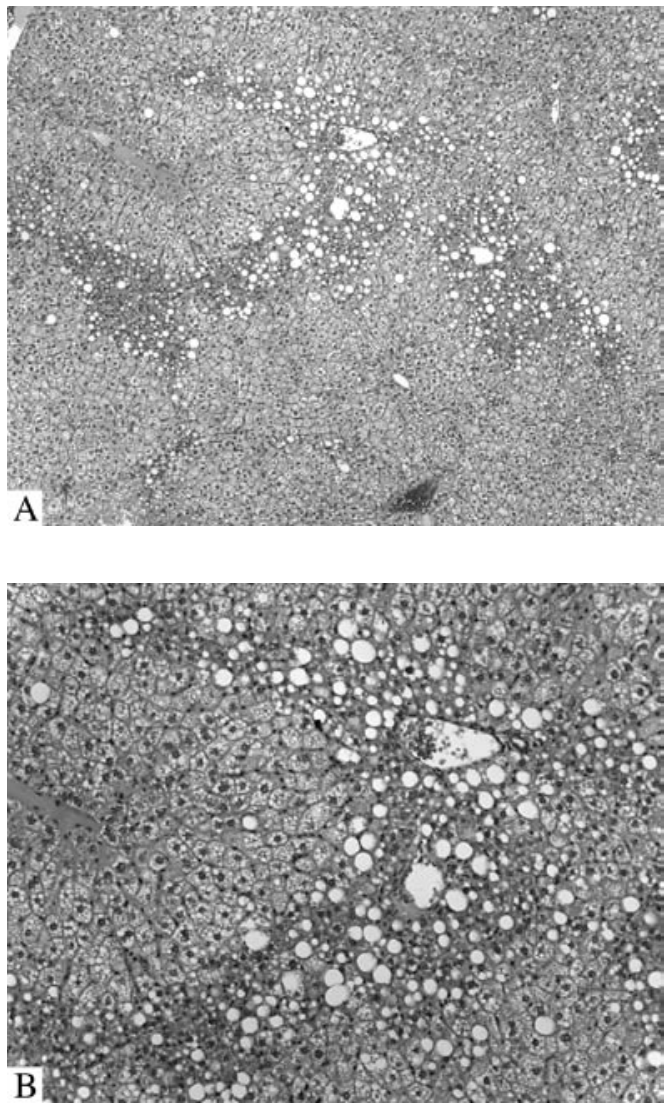

Figure 4. Massive diffuse and microscopic evidence of liver steatosis observed in untreated animals fed a Western diet; (A) x20 and (B) x40 magnification.

Moreover, the inflammatory infiltrate surrounding the plaque was reduced in treated (PNP) mice vs. untreated ones.

\section{Discussion}

Atherosclerotic lesions (atheromata) are asymmetrical focal thickenings of the innermost layer of the artery, the intima. They consist of cells, connective-tissue elements, lipids, and debris. Blood-borne inflammatory and immune cells constitute an important part of an atheroma, the remainder being vascular, endothelial and smooth-muscle cells. The atheroma is preceded by an accumulation of lipid-laden cells beneath the endothelium. Most of these cells in the fatty streak are macrophages, together with some T-cells. In the centre of an atheroma, foam cells and extracellular lipid droplets form a core region, surrounded by a cap of smooth-muscle cells and a collagen-rich matrix. T-cells, macrophages, and mast cells infiltrate the lesion and are particularly abundant in the shoulder region where the atheroma grows. Many of the immune cells exhibit signs of activation and produce inflammatory cytokines, such as IL-1, IL-6 and TNF $\alpha$ (1-3).

There are two major causes of coronary thrombosis, plaque rupture and endothelial erosion. Plaque rupture exposes prothrombotic material from the core of the plaque, phospholipids, tissue factor, and platelet-adhesive matrix molecules, to the blood.

A T-cell infiltrate is always present in atherosclerotic lesions (1-3). T-lymphocytes play critical roles in the pathogenesis of 
atherosclerosis through production of EGF and bFGF (6), numerous inflammatory molecules and proteolytic enzymes able to activate cells in the core, transforming the plaque into an unstable structure that can rupture, induce a thrombus, and elicit an acute coronary syndrome.

Mature plaques (atheromas) have, in the centre, foam cells and extracellular lipid droplets form a core region that is surrounded by a cap of smooth muscle cells and a collagen-rich matrix. Other cell types present in plaques include dendritic cells (DCs), mast cells, a few B cells and probably natural killer T (NKT) cells. The shoulder region of the plaque, which is where it grows, and the interface between the cap and the core present particularly abundant accumulations of T-cells and macrophages (1-3). Many of these immune cells show signs of activation and produce pro-inflammatory cytokines such as IFN $\gamma$. They may progress into a more complex lesion, the lipid core, which is a paucicellular pool of cholesterol deposits surrounded by a fibrous cap. The fibrous cap prevents contact between the blood and the pro-thrombotic material in the lesion. Disruption of the cap leads to thrombosis and many of the adverse clinical outcomes associated with atherosclerosis. Additionally, IFN $\gamma$ inhibits endothelial-cell proliferation, the proliferation and differentiation of vascular smooth muscle cells, and also decreases the production of collagen by these smooth muscle cells. Decreasing the cell and collagen content of the fibrous cap may reduce the stability of the plaque. Therefore, the combined effects of IFN $\gamma$ on cells of the forming plaque promote inflammation and extracellular-matrix destabilisation (1-3).

Heparin is a glycosaminoglycan composed of chains of alternating residues of D-glucosamine and uronic acid. Its major anticoagulant effect is accounted for by a unique pentasaccharide with a high affinity binding sequence to antithrombin III, resulting in inhibition of activated Factor-Xa and thrombin (14). Heparin also binds to endothelial cells and macrophages, a property that contributes to its complicated pharmacokinetics (14-16). Interestingly, heparins have been shown to inhibit the production of pro-inflammatory cytokine and integrins by LPS- or interferon-c-stimulated human monocytes, even when heparin is added after stimulation $(17,21)$, and actively reduce the process of leukocyte recruitment into the site of injury.

Heparin appears to have a greater potential than any other agent tested to neutralize the atherogenic process throughout most of its stages. This potential is supported by the success of clinical trials in which heparin was administered by intravenous, subcutaneous and intrapulmonary routes $(15,16)$. The LMWH are a new class of anticoagulants that have been developed for clinical use. They are fragments of heparin, produced by either chemical or enzymatic depolymerization. Owing to their shorter lengths, LMWH are less able to bridge anti-thrombin and thrombin, resulting in less thrombin inhibition, but they demonstrate a longer half-life, better bioavailability at low doses and less bleeding than UFH (14).

Parnaparin, (24-28) is the sodium salt of a LMWH that is obtained by radical-catalyzed depolymerization of heparin, using hydrogen peroxide and cupric salt, from porcine intestinal mucosa. The majority of the components have a 2-O-sulpho$\alpha$-L-idopyranosuric acid structure at the non-reducing end and a 2-N,6-O-disulpho-D-glucosamine structure at the reducing end of their chain (29). Parnaparin is an LMWH which, like other members of its class, apparently demonstrates a greater antithrombotic effect attributable to its anticoagulant activity when compared with UFH (heparin) from which it is derived. Moreover, subcutaneous Parnaparin has a greater bioavailability and longer half-life than heparin, permitting once-daily administration for the prophylaxis of deep venous thrombosis (DVT) or the treatment of established vascular disorders. The risk of general bleeding appears to be similar with Parnaparin or heparin, although Parnaparin results in fewer haematomas at the site of injection, partly because of the less frequent administration regimen. Compared with heparin, Parnaparin offers the advantages of a more convenient administration regimen coupled with improved local tolerability. (28). We decided to investigate the possible correlation between PNP and the control of the atherogenic process, using ApoE-/- mice maintained on a standard and a Western diet. In both models we observed the development of liver steatosis, which was massive with the Western diet, and moderate, when mice were fed the standard diet. After the subcutaneous administration of Parnaparin in both groups of mice, we observed a quite moderate liver steatosis in Parnaparin-treated animals, which displayed microsteatosis, in comparison to the massive and diffuse steatosis observed in the control animals (untreated). Additionally, in the control (untreated) animals, we found marked atherosclerotic lesions in the aortic wall, with choleterol plaques in the sub-endothelial layer together with an inflammatory infiltrate with macrophages and lymphocytes around the lesion. On the contrary, in treated animals (PNP) the atherosclerotic plaques were reduced in size in the inflammatory infiltrate, suggesting a direct role of Parnaparin in regulating the development of the atherogenic process.

Therefore, Parnaparin seems to be efficient in slowing down the inflammation process, important in the evolution of atherosclerosis and endothelial erosion, probably influencing the recruitment of immune cells and platelet aggregation, and slowing down liver steatosis due to hypercholesterolemia present in ApoE-/- mice. These encouraging data suggest the possibility of testing Parnaparin in clinical trials, as a new adjuvant therapy in atherogenic and thrombotic diseases.

\section{References}

1. Packard RR and Libby P: Inflammation in atherosclerosis: from vascular biology to biomarker discovery and risk prediction. Clin Chem 54: 24-38, 2008.

2. Hansson GK: Mechanisms of disease: inflammation, atherosclerosis, and coronary artery disease. N Engl J Med 352: 1685-1695, 2005.

3. Hansson GK and Libby P: The immune response in atherosclerosis: a double-edged sword. Nature 6: 508-519, 2006.

4. Dahl TB, Yndestad A, Skjelland M, et al: Increased expression of visfatin in macrophages of human unstable carotid and coronary atherosclerosis: possible role in inflammation and plaque destabilization. Circulation 115: 972-980, 2007.

5. Frostegard J, Ulfgren AK, Nyberg P, et al: Cytokine expression in advanced human atherosclerotic plaques: dominance of proinflammatory (Th1) and macrophage-stimulating cytokines. Atherosclerosis 145: 33-43, 1999.

6. Tedgui A and Mallat Z: Cytokines in atherosclerosis: pathogenic and regulatory pathways. Physiol Rev 86: 515-581, 2006.

7. Szodoray P, Timar O, Veres K, et al: TH1/TH2 imbalance, measured by circulating and intracytoplasmic inflammatory cytokines - immunological alterations in acute coronary syndrome and stable coronary artery disease. Scand J Immunol 64: 336-344, 2006. 
8. Tuomisto K, Jousilahti P, Sundvall J, et al: C-reactive protein, interleukin-6 and tumor necrosis factor alpha as predictors of incident coronary and cardiovascular events and total mortality. A population-based, prospective study. Thromb Haemost 95: $511,2006$.

9. Yamagami H, Kitagawa K, Hoshi T, et al: Associations of serum IL-18 levels with carotid intima-media thickness. Arterioscler Thromb Vasc Biol 25: 1458-1462, 2005.

10. Elkind MS, Rundek T, Sciacca RR, et al: Interleukin-2 levels are associated with carotid artery intima-media thickness. Atherosclerosis 180: 181-187, 2005.

11. Heeschen C, Dimmeler S, Hamm CW, et al: Serum level of the antiinflammatory cytokine interleukin-10 is an important prognostic determinant in patients with acute coronary syndromes. Circulation 107: 2109-2114, 2003.

12. Gielen S, Sandri M, Schuler G and Teupser D: Risk factor management: antiatherogenic therapies. Eur J Cardiovasc Prev Rehabil 16 (Suppl. 2): S29-S36, 2009.

13. Dobarro D, Gómez-Rubín MC, Sanchez-Recalde A, Moreno R Galeote G, Jimenez-Valero S, Calvo L, de Sá EL and LópezSendón JL: Current pharmacological approach to restore endothelial dysfunction. Cardiovasc Hematol Agents Med Chem 7: 212-222, 2009.

14. Hochart H, Jenkins PV, Smith OP and White B. Low-molecular weight and unfractionated heparins induce a downregulation of inflammation: decreased levels of proinflammatory cytokines and nuclear factor-kappaB in LPS-stimulated human monocytes Br J Haematol 133: 62-67, 2006.

15. Kanabrocki EL, Sothern RB, Bremner WF, et al: Heparin as a therapy for atherosclerosis: preliminary observations on the intrapulmonary administration of low-dose heparin in the morning versus evening gauged by its effects on blood variables. Chronobiol Int 8: 210-233, 1991.

16. Kanabrocki E: A quest for the relief of atherosclerosis: potential role of intrapulmonary heparin - a hypothesis. Q J Med 83 . 259-282, 1992

17. Attanasio M, Gori AM, Giusti B, Pepe G, Comeglio P, Brunelli T, Prisco D, Abbate R, Gensini GF and Neri Serneri GG. Cytokine gene expression in human LPS- and IFNgamma-stimulated mononuclear cells is inhibited by heparin. Thromb Haemost 79: 959-962, 1998.

18. Høgåsen $\mathrm{AK}$ and Abrahamsen TG: Heparin suppresses lipopolysaccharide-induced monocyte production of several cytokines, but simultaneously stimulates $\mathrm{C} 3$ production. Thromb Res 80 : 179-184, 1995
19. Gori AM, Attanasio M, Gazzini A, Rossi L, Lucarini L, Miletti S, Chini J, Manoni M, Abbate R and Gensini GF: Cytokine gene expression and production by human LPS-stimulated mononuclear cells are inhibited by sulfated heparin-like semisynthetic derivatives. J Thromb Haemost 2: 1657-1662, 2004.

20. Manduteanu I, Voinea M, Antohe F, Dragomir E, Capraru M, Radulescu L and Simionescu M: Effect of enoxaparin on high glucose-induced activation of endothelial cells. Eur J Pharmacol 477: 269-276, 2003.

21. Hecht I, Hershkoviz R, Shivtiel S, Lapidot T, Cohen IR, Lider O, and Cahalon L: Heparin-disaccharide affects T cells: inhibition of NF-kappaB activation, cell migration, and modulation of intracellular signaling. J Leukoc Biol 75: 1139-1146, 2004.

22. Maugeri N, de Gaetano G, Barbanti M, Donati MB and Cerletti C: Prevention of platelet-polymorphonuclear leukocyte interactions: new clues to the antithrombotic properties of parnaparin, a low molecular weight heparin. Haematologica 90: 833-839, 2005

23. McKeage $\mathrm{K}$ and Keating GM: Parnaparin: a review of its use in the management of venous thromboembolism, chronic venous disease and other vascular disorders. Drugs 68: 105-122, 2008.

24. Marchi EG: Alfa Wassermann low molecular weight heparin. In: Low Molecular Weight Heparins in Clinical Practice. Doutremepuich C (ed.), Marcel Dekker Inc. New York, Basel, Hong Kong, pp237-244, 1992.

25. Dettori AG: Parnaparin: a review of its pharmacological profile and clinical application. Drugs Today 31: 19-35, 1995.

26. Dettori AG and Babbini M: Human pharmacology of a low molecular weight heparin (Alfa-LMWH): an update. Med Res Rev 12: 373-389, 1992.

27. Macchi M and Maggiore L: OP-2123. Drugs Future 12: 1111-1112, 1987.

28. Frampton JE and Faulds D: Parnaparin . A review of its pharmacology and clinical application in the preventive and treatment of thromboembolic and other vascular disorders Drugs 47: 652-676, 1994.

29. Parnaparin sodium. In: European Pharmacopoiea. 5th edition, Council of Europe, European Directorate for the Quality of Medicines, Strasbourg, 2005. 